\title{
Applications of Wavelet Packets Decomposition in Iris Recognition
}

\author{
Junying Gan and Yu Liang \\ School of information, Wuyi University, \\ Jiangmen, Guangdong, P.R.C. 529020 \\ jygan@wyu.cn
}

\begin{abstract}
The method of Wavelet Packets Decomposition (WPD) originating from wavelet transform is more accurate in signal analysis, with the predominance of analyzing high-frequency information. Combined with the trait of WPD, an algorithm for iris recognition is presented in this paper. Firstly, iris image is divided into several windows, and WPD is done to them. At the same time, some of the subband images from each window are selected, which contain most information of iris image. Secondly, the farther feature extraction and compression are applied to these subband images by way of Singular Value Decomposition (SVD), and iris recognition features are obtained. Finally, Weighted Euclidean Distance (WED) classifier is utilized in recognition. Experimental results on CASIA (Chinese Academy of Sciences, Institute of Automation) iris image database show the method is valid in iris recognition.
\end{abstract}

\section{Introduction}

Iris recognition has the best potential of development and the bright future due to its advantages of invariability, stability, acquirability, un-intrusion and so on, though its history is about 20 years. Statistical data reveals that compared with face, voice and other untouched method of identity authentication, iris has higher veracity and becomes a hot study field these years [1]. Daugman is the one who first studied iris recognition, and proposed the method of iris texture phase encoding based on Gabor wavelet [2]. Wildes presented the approach for decomposing iris image using Gaussian filter at different resolution level [3]. Boles introduced a zero-crossing detection way based on wavelet transform [4]. All these methods are based on wavelet transform. Development of iris recognition technology was accompanied by the application of wavelet transform altogether. In recent years, the method of Wavelet Packets Decomposition (WPD) gradually draws attention. Chen ji et al investigated a quality evaluation method of iris image based on WPD [5]. Emine Krichen et al contrasted the recognition results of iris image acquired under the conditions of visible light by a standard camera with ones under the conditions of near infrared illumination by a monochrome CCD camera. He verified that under the conditions of visible light illumination, the use of classical wavelet transform is not so satisfactory compared with WPD [6]. 
Iris possesses abundant detailed information in texture, and one of the key problems in iris recognition is how to extract iris features effectively. It is well known high-frequency information can be analyzed by WPD. Integrated with the trait of WPD, an approach for iris recognition based on WPD is presented in this paper. Firstly, division is done to each iris image, and several windows with the same size are acquired. Secondly, WPD is applied to these windows, and then a set of subband images from each window are gained. In order to extract iris features adequately, some of subband images from each window need be selected. For making farther feature extraction and compression, Singular Value Decomposition (SVD) is applied in the selected subband images with the help of the properties of Singular Value (SV). Finally, Weighted Euclidean Distance (WED) classifier is used in iris recognition. Experimental results on CAISA (Chinese Academy of Sciences, Institute of Automation) iris image database show that, correct recognition rate in this paper reaches $100 \%$ and a new approach in iris recognition is supplied.

\section{Wavelet Packets Decomposition}

WPD originating from wavelet transform is more accurate in signal analysis. Wavelet transform is one kind of mathematical tool, which is used for function decomposition in multilevel. After wavelet transform, a signal can be described by its wavelet coefficients. Because of this, wavelet transform becomes more and more attractive in the fields of texture recognition, signal processing, image processing, pattern recognition and so on.

In the process of wavelet transform, wavelet function $\psi(x)$ called wavelet base is introduced, which is made $b$ translation and then convolved with $f(x)$ analyzed in different scale $a$. That is

$$
\mathrm{W} \mathrm{T}_{f}(a, b)=\frac{1}{\sqrt{a}} \int_{-\infty}^{\infty} f(x) \overline{\psi\left(\frac{x-b}{a}\right)} d x, \quad a>0
$$

Two-dimensional wavelet transform can be obtained by the generalization of onedimensional wavelet transform. $\mathrm{WT}_{f}(a, b)$ is regarded as wavelet transform only in direction $x$. On the basis of it, wavelet transform in direction $y$ is introduced, in which $b$ and $c$ denote translation parameters in direction $x$ and direction $y$ respectively. Here, $\psi\left(\frac{x-b}{a}\right)$ is defined as wavelet function in direction $x$; whereas $\psi\left(\frac{y-c}{a}\right)$ is wavelet function in direction $y$. Suppose $f(x, y)$ express twodimensional signal analyzed, two-dimensional wavelet transform can be deduced as

$$
\mathrm{WT}_{f}(a, b, c)=\frac{1}{\sqrt{a}} \int_{R} \int_{R} f(x, y) \overline{\psi_{x}\left(\frac{x-b}{a}\right) \psi_{y}\left(\frac{y-c}{a}\right)} \mathrm{d} x \mathrm{~d} y
$$

In image processing, binary wavelet is usually used. Suppose scales are discretized as the power of 2 , which is expressed as $a=2^{0}, 2^{1}, 2^{2}, \cdots, 2^{j}, j=1,2, \cdots, N$. Tiny change of $j$ can result in a great change in scale, and it shows that binary 
wavelet is an effective strategy from coarse to fine signal analysis. If $f(x, y)$ represents iris image, two-dimensional wavelet transform can be done respectively by different one-dimensional filters in direction $x$ and direction $y$. Then a set of subband images in low and high frequency can be gained, as shown in Fig.1(a), where $L L$ represents horizontal and vertical low-frequency information, $H L$ horizontal highfrequency and vertical low-frequency information, $L H$ horizontal low-frequency and vertical high-frequency information, $H H$ horizontal high-frequency and vertical high-frequency information which is named as diagonal detail part. Through wavelet transform, the more evident image information features in one frequency or direction are, the more powerful the energy of the corresponding subband image will be. That is to say, features of iris image concentrates mainly on a few wavelet coefficients.

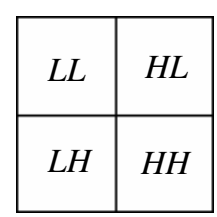

(a)

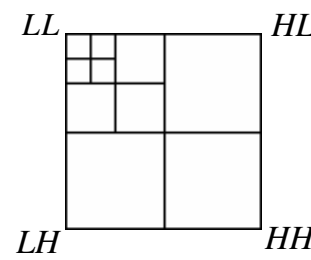

(b)

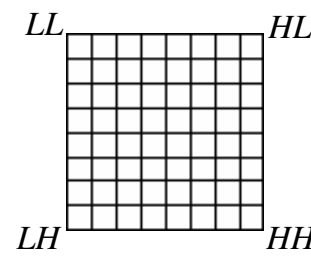

(c)

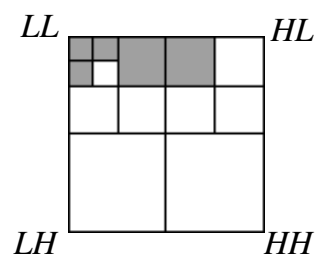

(d)

Fig. 1. Wavelet transform and WPD of iris image

In practical application, a signal can be divided into low-frequency and highfrequency information by wavelet analysis. Low-frequency information describes approximation part; whereas high-frequency information describes detail part. Then, decomposition is done to the approximation part. Thus its approximation and detail parts can be gained again. This operation can happen to the approximation part frequently if necessary, but never to the detail part, as shown in Fig.1(b). While each subband is divided into multilevel by WPD, it's more powerful in signal description and becomes compensation for wavelet analysis insufficiency in highfrequency information. In a word, decomposition is not only done to the approximation part, but also to the detail part, as shown in Fig.1(c). When WPD is used in texture image analysis, besides the analysis of approximation part, orthogonal decomposition is done to the selected detail part, and most information of iris image can be gained.

\section{Wavelet Packets Decomposition in Iris Recognition}

The flow char on WPD in iris recognition is shown in Fig.2. A series of operations to the acquired iris image are iris image processing, image division, WPD of windows, subband images selection, SVD and SV compression (SVC), and WED classifier respectively. The procedures of iris image processing and WPD in iris feature extraction will be discussed detailed. 


\subsection{Iris Image Preprocessing}

Iris image preprocessing includes localization, normalization and image enhancement. Iris shape is approximately annular, in which inner round refers to the boundary between pupil and iris, and outer round refers to the boundary between iris and sclera. Therefore, localization of inner and outer round of iris image need be done respectively with two rounds un-homocentric. Firstly, threshold is utilized to pupil segmentation, then erosion and dilation are done to eliminate the useless spots, and projection is taken to carry out inner round localization, as shown in Fig.3(a). Because the gray between iris and sclera is not so evident, threshold method cannot be used to fix the two boundaries again. In this paper, Hough transform is utilized to iris outer round localization, in which Gaussian filter is used to iris image for smoothness firstly, then Canny operator is successively processed to the image for edge detection, and the outline of iris image is attained. According to the localization results of iris inner round, parameter ranges in Hough transform are limited. The center and radius of iris outer round can be fixed by Hough transform, as shown in Fig.3(a).

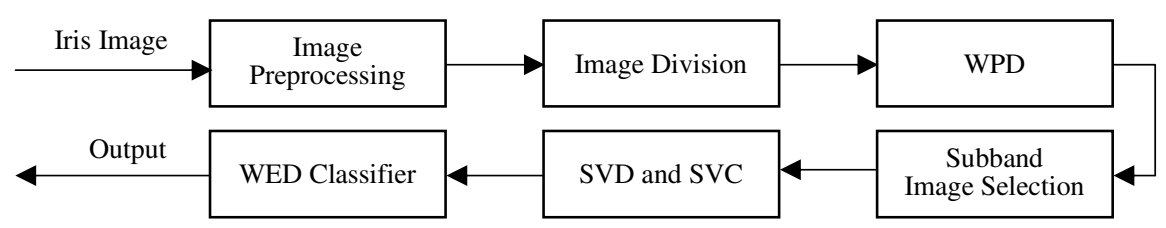

Fig. 2. Flow chart on iris recognition

To eliminate the influence of translation, proportion and rotation, it is necessary to adjust iris image to the same size after localization. Normalization is to map each pixel of iris image from orthogonal coordinates to polar coordinates, denoted by $I(x(r, \theta), y(r, \theta)) \rightarrow I(r, \theta)$, where $I(x, y)$ refers to the iris image after localization, and $I(r, \theta)$ the representation of iris image in polar coordinates. After normalization, iris image possesses translation invariance and changing invariance of iris inner and outer circle size. And iris image becomes a $64 \times 512$ rectangle in polar coordinates. For increasing recognition rate and lessening the influence of illumination, image enhancement is done to the iris rectangle by histogram equalization, as shown in Fig.3(b). Fig.3(b) demonstrates eyelid and eyelashes envelop iris partly, and can decrease recognition rate if feature extraction is done to the iris rectangle directly. In this paper, part of the enveloped iris is discarded, and a $64 \times 256$ iris rectangle is acquired as the object of feature extraction, as shown in Fig.3(c).
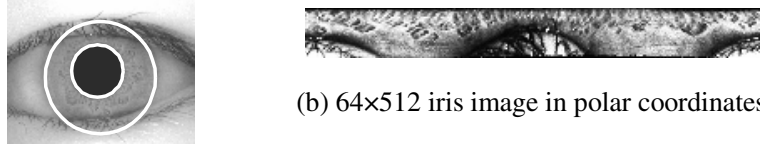

(b) $64 \times 512$ iris image in polar coordinates

(a) Iris image localization

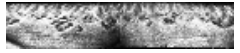

(c) $64 \times 256$ iris image

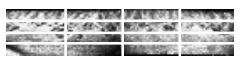

(d) Iris image division

Fig. 3. Iris image preprocessing 


\subsection{Iris Feature Extraction by WPD}

Iris image contains abundant information of texture details. If WPD is done to the whole iris image directly, a series of wavelet coefficients can be obtained. If the energy and variance of wavelet coefficients are regarded as iris image features, a great deal of image information can be abandoned and noise in high-frequency detail part can also be introduced. All these aspects can affect iris recognition rate. In order to extract local detail part of iris texture adequately, iris image is first divided into several windows with the same size, as shown in Fig.3(d). In Fig.3(a), the distribution of iris texture is radial. When iris image is mapped to polar coordinates, the distribution of iris texture is vertical. After done by wavelet transform, high-frequency information of iris image concentrates on horizontal detail part $H L$. While $H H$ representing diagonal high-frequency information of iris image contains much noise, it is not suitable for feature extraction. In the process of wavelet transform, if the resolution levels are not enough, it can result in deficiency in classified information; whereas too many resolution levels can lead to huge calculations and the boosting up of boundary impact owing to small subbands. These aspects can affect iris recognition rate. Therefore, in this paper, Daubechies- 4 wavelet decomposition is adopted, and each window of iris image is divided into 3 levels. After that, $H L_{1}$ containing most high-frequency information, whose subscript means sequence number of decomposition levels, is further decomposed according to the theory of WPD, and the corresponding low-frequency and high-frequency information are gained. In order to maintain effective information, get rid of the redundancy and diminish the effect of noise, $L L_{3}, H L_{3}, L H_{3}, H L_{2}$ and $H L_{1-} L L$ altogether 5 subband images are held as the feature extraction objects, as shown in the shadows of Fig.1(d).

After WPD, SVD is applied to these 5 subband images respectively, and $5 \mathrm{SV}$ vectors are acquired. Then, compression of SV vectors is necessary on account of redundant information and image noise. Reference [7] shows that the larger elements of SV play the key role in recognition. Therefore, the larger ones are maintained and the smaller ones omitted, which can help not only hold most information of iris image, but also weaken the influence of noise. In addition, the number of recognition features can be lessened greatly. Afterwards, the final recognition features are obtained, and WED classifier is utilized in iris recognition.

\section{Experimental Results and Analysis}

In this paper, experiments have been done on CASIA iris image database offered by the National Laboratory of Pattern Recognition (NLRP). This database (version 1.0) includes 756 iris images from 108 eyes of 80 subjects. They are gray images with resolution $320 \times 280$. For each eye, 7 images are captured in two sessions, where three samples are collected in the first session and the other four in the second session. In this paper, 280 images with 40 classes in CASIA iris image database are randomly selected in experiments.

In the process of image division, horizontal and vertical directions are divided into 4 and 4 respectively. So 16 windows are obtained, as shown in Fig.3(d). WPD is based on 3-level wavelet transform. First, All windows are processed respectively by 
Daubechies-4 wavelet transform in 3 levels. After that, Daubechies-4 wavelet decomposition is utilized again to $H L_{1}$ where iris texture high-frequency detail part concentrates, and $H L_{1-} L L$ retaining rich information of $H L_{1}$ is kept. Then $L L_{3}$, $H L_{3}, L H_{3}, H L_{2}$ and $H L_{1-} L L 5$ subband images of each window are chosen as the objects of further feature extraction, as shown in the shadows of Fig.1(d).

$L L_{3}, H L_{3}$, and $L_{3}$ are three matrixes consisting of wavelet coefficients with the same size $8 \times 14$; whereas $H L_{2}$ and $H L_{1-} L L$ are $9 \times 21$. Next, [ $L L_{3} H L_{3} L_{3} H L_{2}$ $\left.H L_{1-} L L\right] 5$ subband images are processed apart by SVD to obtain $5 \mathrm{SV}$ vectors, the elements number of each $\mathrm{SV}$ vector are respectively $\left[\begin{array}{lllll}8 & 8 & 8 & 9 & 9\end{array}\right]$. With view of redundant information and noise contained in the SV vectors and the un-practicality of too many features, compression is done to the SV vectors. That is to preserve the larger elements of each SV vector, and then reshape the $5 \mathrm{SV}$ vectors to a onedimensional recognition feature vector. Finally, WED classifier is used in recognition.

Table 1 shows iris recognition rate based on WPD. In the experiments, the elements of $5 \mathrm{SV}$ vectors are selected respectively in different ways. The number of training and testing samples is also changed to gain the results of iris recognition. In the meanwhile, several front images of each iris class are chosen as training samples, and the remainder ones of each iris class as testing samples. From Table 1, it can be known that, when the larger elements number of SV vectors corresponding to the

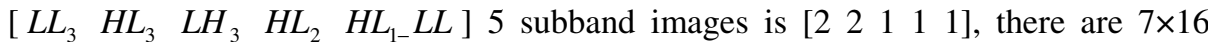
feature elements with 112 in total, and the number selection of training and testing samples is 6_1, recognition rate reaches $100 \%$. At the same time, increasing or decreasing the elements of $\mathrm{SV}$ vectors both results in the descending of recognition rate. But decreasing training samples and increasing testing samples results in the descending of recognition rate. When the number selection of training and testing samples is 3_4, recognition rate is $88.13 \%$. On the whole, the selection of SV vectors in this case has better performance than the ones in other case. At the same time, increasing or decreasing the elements of SV vectors both results in the descending of recognition rate.

Table 1. Correct recognition rate of iris recognition on WPD

\begin{tabular}{|c|c|c|c|c|}
\hline$\left[L L_{3} H L_{3} L H_{3} H L_{2} H L_{1-} L L\right] \times 16$ & $6 \_1$ & $5 \_2$ & $4 \_3$ & 3_4 \\
\hline$\left[\begin{array}{lllll}3 & 3 & 2 & 1 & 1\end{array}\right] \times 16=160$ & $95 \%$ & $95 \%$ & $93.33 \%$ & $90 \%$ \\
\hline$\left[\begin{array}{lllll}3 & 3 & 1 & 2 & 1\end{array}\right] \times 16=160$ & $97.5 \%$ & $92.5 \%$ & $91.67 \%$ & $86.88 \%$ \\
\hline$\left[\begin{array}{lllll}3 & 3 & 1 & 1 & 1\end{array}\right] \times 16=144$ & $97.5 \%$ & $95 \%$ & $92.5 \%$ & $88.75 \%$ \\
\hline$\left[\begin{array}{lllll}3 & 2 & 1 & 1 & 1\end{array}\right] \times 16=128$ & $100 \%$ & $95 \%$ & $92.5 \%$ & $88.13 \%$ \\
\hline$\left[\begin{array}{lllll}2 & 2 & 1 & 1 & 1\end{array}\right] \times 16=112$ & $100 \%$ & $95 \%$ & $92.5 \%$ & $88.13 \%$ \\
\hline$\left[\begin{array}{lllll}2 & 1 & 1 & 1 & 1\end{array}\right] \times 16=96$ & $100 \%$ & $95 \%$ & $90 \%$ & $85.63 \%$ \\
\hline$\left[\begin{array}{lllll}1 & 1 & 1 & 1 & 1\end{array}\right] \times 16=80$ & $95 \%$ & $93.75 \%$ & $87.5 \%$ & $81.25 \%$ \\
\hline
\end{tabular}

*: The number ' 6 ' shown in ' $6 \_1$ ' denotes the number of training samples in iris recognition, and ' 1 ' denotes the number of testing samples, the same to the others. 
In addition, image division can also influence recognition rate. In this paper, the division of $4 \times 4$ is better than the other way of divisions. It is due to the proper consideration of local iris detail part in the vertical direction. If image is divided into more windows, the whole distribution of iris texture can be out of consideration.

\section{Conclusions and Outlook}

With the predominance of analyzing high-frequency information, WPD is utilized in iris image in this paper. Being a good algebraic description tool, SVD is applied to subband image, which contains most of iris texture detail part. Then SV compression is done to acquire final recognition features. Experimental results demonstrate that, the approach of iris recognition based on WPD is valid. But recognition rate of the approach is influenced by SV compression. Larger or smaller compression range can also result in the descending of iris recognition. Therefore, it's necessary to make further study on iris feature extraction and recognition.

\section{Acknowledgement}

This work is supported by NSF of Guangdong Province, P.R.C. (No.032356).

\section{References}

1. Anil K Jain, et al: Biometrics Personal Identification in Networked Society[M]. 101 Philip D river, A ssinippi Park, Norwell, Massachusetts 02061 USA, Kluwer Academic Publishers (1999) 103-121

2. Daugman J: High confidence visual recognition of persons by a test of statistical independence[J]. IEEE Trans. Pattern Analysis and Machine Intelligence, Vol. 15, No. 11, (1993)1148-1161

3. Woggen U: Optical properties of semiconductor quantum dots[M]. Germany: Springer, (1996)179-185

4. Bobles W W: A human identification technique using image of the iris and wavelet transform[J], Vol. 46. IEEE Transaction on Signal Processing (1998)1185 -1188

5. Chen Ji, Hu Guangshu, XU Jin: Iris image quality evaluation method based on wavelet packets decomposition[J], Vol.43, No.3. J Tsinghua Univ (Sci Tech)(2003)377-380

6. Emine Krichen, M. Anouar Mellakh, Sonia Garcia-Salicetti, Bernadette Dorizzi: Iris Identification Using Wavelet Packets[C], Vol. 4. Pattern Recognition, 17th International Conference, Proceeding of ICPR'04(2004)335-338

7. Gan Junying, Zhang Youwei: A New Approach for Face Recognition Based on Singular Value Features and Neural Networks[J], Vol. 32, No. 1. Acta Electronica Sinica(2004) 170-173

8. Yunhong Wang, Yong Zhu, Tieniu Tan: Biometrics Personal Identification Based on Iris Pattern[J], Vol. 28, No, 1. Acta Automatica Sinics(2002)1-10

9. Qing Qianqing, Yang Zongkai: Wavelet Analysis in Practical Applications[M]. Xi Dian Univerity Press (1998) 